\title{
Biochemical changes as a result of prolonged strenuous exercise
}

\author{
D E R Warburton, R C Welsh, M J Haykowsky, D A Taylor, D P Humen
}

Br J Sports M ed 2002;36:301-303

Objective: To briefly review biochemical changes that may result from prolonged strenuous exercise and to relate these changes to health risk.

Methods: Medline and Sports Discus databases were searched for relevant articles. Additional articles were found using cross referencing and the authors' knowledge of the subject area.

Results: Prolonged strenuous exercise may result in a series of biochemical changes that are of concern from a health point of view. G enerally, these changes are benign, but some, especially hyponatraemia, are potentially life threatening occurrences.

Conclusion: Doctors and athletes should be aware of the potentially adverse biochemical changes, especially hyponatraemia, that may result from prolonged strenuous exercise.

p $\mathrm{n}$ recent years, there has been considerable interest in the performance of prolonged (more than one hour) strenuous exercise (PSE), especially ultra-endurance events such as the I ronman triathlon, and there has been increasing research on the impact of PSE on biochemical and/or haematological status. Haematological disturbances after exercise, such as haemolysis and/or iron deficiency, ${ }^{12}$ are well documented and thought to be of concern for optimal endurance performance, but of little concern from a health perspective. ${ }^{2}$ Of great interest to exercise physiologists and doctors is the finding that exercise induced biochemical disturbances may be of concern from both points of view. ${ }^{34}$ Accordingly, the purpose of this report is to provide a brief synopsis of the potential adverse effects of PSE from a health perspective.

\section{METHODS}

The primary database was obtained by a computerised search of M edline and SportDiscus for articles from J anuary 1980 to December 2001. Additional articles were found using cross referencing and the authors' knowledge of the subject area. Owing to the brief nature of this report, only articles on biochemical changes observed after PSE were included.

\section{FINDINGS AND DISCUSSION}

The following sections briefly summarise the literature on the impact of PSE on a wide range of biochemical variables that may have an impact on health status.

\section{Magnesium}

Magnesium is a major cation involved in a series of metabolic pathways that are challenged during exercise. ${ }^{5}$ Exercise may increase the demand for magnesium and/or increase magnesium loss, potentially leading to hypomagnesaemia, which can result in muscle weakness, neuromuscular dysfunction, and tetany, ${ }^{56}$ all of which can affect physical performance and/or health status.
PSE, especially under hot conditions, may lead to hypomagnesaemia. Reductions in serum or plasma magnesium of 5-25\% have been reported immediately after PSE, such as a marathon, ${ }^{6-8}$ a half I ronman triathlon, ${ }^{9}$ prolonged cross country skiing, ${ }^{10} 90$ minutes of treadmill exercise, ${ }^{11}$ and a $120 \mathrm{~km}$ march. ${ }^{12}$ The hypomagnesaemia seems to be greater in a hot climate. ${ }^{11}$ Generally, it is transient, returning to baseline levels after a period of recovery (within 12-72 hours). ${ }^{69}$ However, others have reported magnesium deficiency three months after a $120 \mathrm{~km}$ hike, suggesting total body magnesium depletion. ${ }^{12}$

A small portion of the changes in magnesium caused by PSE may be related to losses in sweat and urine, but most are due to other factors. ${ }^{6810} \mathrm{~A}$ transient shift of magnesium to the intracellular space during exercise is a probable explanation for a large proportion of the hypomagnesaemia. ${ }^{6}$ Golf et $a^{13}$ postulated that, as the exercise duration increases, the magnesium will shift from an erythrocyte reservoir into the plasma and then to the working muscles. With prolonged exercise (more than one hour), hypomagnesaemia may occur as a result of the depletion of the erythrocyte reservoir. ${ }^{13}$ Other investigators have also postulated that, as exercise is prolonged and fatty acid metabolism is increased, magnesium can be taken up into adipocytes, thereby reducing the vascular magnesium concentration. ${ }^{6}{ }^{8}$ Vascular magnesium concentration is thought to be inversely related to free fatty acids. ${ }^{8}$ After completion of the exercise, lipolysis will decrease and magnesium will be released from the adipocytes, allowing plasma magnesium levels to return to normal. ${ }^{56} \beta$-Adrenergic stimulation may also contribute to the uptake of magnesium into adipose cells ${ }^{5}$ as a result of the induction of lipolysis. However, investigators ${ }^{8}{ }^{9}$ have found no significant correlation between catecholamine levels and magnesium depletion after a prolonged endurance event.

Overt signs and symptoms of hypomagnesaemia-for example, hyperirritability, tetany, convulsions, and cardiac arrhythmias - may not be manifested until the serum magnesium concentration has decreased below $0.5 \mathrm{mmol} / \mathrm{l}$. Generally, exercise induced hypomagnesaemia does not approach a level that would be of concern from a health point of view. Also, it is generally transient. Thus, the effect of low magnesium levels caused by PSE on health status in healthy people with normal magnesium levels seems to be negligible.

However, it may be greater in people who already have low serum magnesium levels before PSE in hot environments. ${ }^{8}$ This problem may be exacerbated in those who experience large increases in plasma free fatty acids during PSE, because of the inverse relation between serum magnesium and free fatty acids. The relation between serum fatty acids and magnesium may explain some of the problems observed after PSE. ${ }^{8}$ It is also important to note that magnesium deficiency has been postulated to play a part in myocardial injury after PSE. ${ }^{14}{ }^{15}$ According to Rowe's model, magnesium deficiency may lead to an increased potential for thrombus formation and/or coronary vasospasm, potentially leading to myocardial injury such as myocardial necrosis. This is supported by work 
with the canine model, in which magnesium deficiencies have been associated with increased incidence of coronary vasospasm and sudden death. ${ }^{16}$ Given the potential impact of hypomagnesaemia on health status, careful monitoring of total body magnesium stores may be warranted, especially in athletes who consistently engage in PSE in hot climates.

\section{Potassium}

Potassium, the major cation of the intracellular fluid, is released from muscle cells during exercise in direct relation to exercise intensity. ${ }^{17}{ }^{18} \mathrm{~A}$ rise in potassium (hyperkalaemia) is rapidly reversed after rest from exercise ${ }^{17}{ }^{19}$ and may even be associated with a lowering of potassium levels to below control levels (hypokal aemia). ${ }^{17} 19$

Slight hypokalaemia has been reported immediately after a half Ironman triathlon ${ }^{9}$ and an ultra-triathlon, ${ }^{20}$ whereas others have reported slight hyperkalaemia after a marathon. ${ }^{7} 811$ The hyperkalaemia is thought to be due to an exercise induced shift of potassium from the intracellular to the extracellular space. ${ }^{11}$ The hypokalaemia is generally thought to be due to reuptake of potassium into the muscle after exercise perhaps as the result of the continuation of catecholamine stimulation of the sarcolemmal sodium/potassium ATPase without anaerobic metabolism or muscle ischaemia. ${ }^{18}$ The resultant hypokalaemia may also be due to increased blood flow to the skeletal muscles and/or increased intracellular acidosis. ${ }^{17}$

Exercise induced hyperkalaemia generally has no effect on athletes and may even be attenuated. ${ }^{21}$ However, hyperkalaemia may be associated with dangerous cardiotoxicity ${ }^{21}$ and arrythmogenic events in people with underlying coronary artery disease ${ }^{18}$ and could explain certain instances of sudden cardiac death after PSE. ${ }^{22}$ The exercise induced hypokalaemia may also be associated with arrythmogenic events in people with underlying coronary artery disease. ${ }^{18}$ The physiological significance of the exercise induced potassium changes in healthy people seems to be small. However, this does not rule out the importance of exercise induced potassium changes in sudden cardiac death in people with underlying cardiovascular disease. As athletes who engage in PSE are not all free of cardiovascular disease, doctors and coaches should be aware of the potential dangers of potassium shifts resulting from this form of exercise.

\section{Sodium}

Exercise induced hyponatraemia (sodium $<135 \mathrm{mmol} / \mathrm{l}$ ) is the principal electrolyte disorder seen after prolonged endurance exercise, and is considered by some ${ }^{4}$ to be the greatest risk to athletes engaging in PSE. Sodium is the major cation of the extracellular fluid. A reduction in the extracellular sodium concentration will result in a fluid shift into the intracellular space, which can lead to cellular swelling and its associated complications. Severe hyponatraemia (sodium <130 mmol/l) has been associated with several complications, including mild symptoms, such as malaise, nausea, fatigue, and confusion, ${ }^{3}$ and more severe symptoms, including seizures, respiratory arrest, increased intracranial pressure, coma, and death. ${ }^{3423}$

Symptomatic hyponatraemia has been observed in 0.1$4 \%^{34}$ of athletes engaged in PSE and about $9 \%$ of collapsed ultra-endurance athletes. ${ }^{4}$ The incidence of hyponatraemia in athletes seeking medical attention after PSE has been reported to be as high as $27 \% .{ }^{3}$ It is also important to note that many asymptomatic athletes exhibit low sodium concentrations after PSE, but do not seek medical attention. ${ }^{3}$

The incidence of hyponatraemia seen after PSE increases with the race distance. Hyponatraemia is seldom observed in athletes after four hours of exercise, but is more common in races lasting longer than eight hours. ${ }^{23}$ Therefore, medical personnel should be prepared to treat an increasing proportion of athletes for hyponatraemia as the exercise duration increases.
This problem seems to be more prevalent in recreational athletes who engage in PSE as opposed to highly trained athletes. ${ }^{4}$ Women may also be at a greater risk than men. ${ }^{324}$ Hyponatraemia is not confined to people engaging in competitive athletic events. Symptomatic hyponatraemia has been observed in army trainees and recreational hikers. ${ }^{25}{ }^{26} \mathrm{~A}$ recent investigation has reported the death of an army trainee as a result of complications associated with severe hyponatraemia. ${ }^{25}$

Several possible mechanisms have been postulated to lead to exercise induced hyponatraemia. ${ }^{27}$ Some investigators have speculated that sodium chloride losses in sweat associated with net dehydration may lead to the observed hyponatraemia after PSE. ${ }^{28}{ }^{29}$ However, others have indicated that the severe hyponatraemia observed after PSE is not associated with dehydration..$^{30}$ Also, sodium losses alone are unlikely to account for the observed symptomatic hyponatraemia, ${ }^{27}$ and thus alternative mechanisms must be at play. There is strong evidence that increased dilute fluid intake and retention (in combination with sodium loss in sweat) plays a large part in the development of hyponatraemia. ${ }^{31-33}$ In fact, severe hyponatraemia is commonly associated with fluid overload..$^{31}$ Speedy et $\mathrm{al}^{3}$ recently reported that $73 \%$ of all cases of severe hyponatraemia after the New Zealand I ronman triathlon were the result of fluid overload. Noakes \& $\mathrm{al}^{33}$ observed that athletes engaged in PSE may actually overhydrate with hypotonic solutions, and this, combined with moderate sodium loss through sweat, may make them vulnerable to hyponatraemia and its associated complications. Noakes ${ }^{24}$ postulated that less competitive athletes who consistently take in water during events lasting for at least five hours may be particularly at risk of devel oping symptomatic hyponatraemia.

Given the potential for the development of hyponatraemia and its associated ailments after PSE, doctors should be aware of its prevention and treatment. The simplest prevention measure is to ensure that athletes manage their fluid intake during exercise so that they do not overhydrate. People who perform PSE (whether in the occupational or sport setting) should be made aware of the potentially lethal effects of ingesting fluids in excess of sweat loss. Noakes ${ }^{24}$ has postulated that a fluid intake of about $500 \mathrm{ml} / \mathrm{h}$ is appropriate for most less competitive athletes. Several factors should be considered when treating hyponatraemia. It has been estimated that athletes with symptomatic hyponatraemia are overhydrated by 2-6 litres, and therefore the administration of "uncontrolled fluid replacement" to these athletes has not been advocated. ${ }^{34}$ Several investigators advocate the slow infusion of hypertonic saline (3\%), with careful monitoring of serum sodium concentrations. ${ }^{34}{ }^{35}$ Clinicians dealing with hyponatraemic patients must be aware of the potentially fatal consequences of further fluid overload.

Urea and creatinine

Athletes commonly display high resting urea concentrations, ${ }^{9}$ probably as a result of the continual stress of training. ${ }^{36}$ Urea concentrations are also generally increased after the performance of $\operatorname{PSE}^{96-39}$ and may remain elevated for 24-40 hours after exercise. ${ }^{936}$ An increase in urea concentration may be related to a reduction in renal blood flow (and glomerular filtration rate) secondary to fluid volume deficiency, increased protein catabolism, and/or bleeding into the intestine, all of which may occur after PSE. ${ }^{20} 363839$

Creatinine concentration (the product of creatine breakdown from skeletal muscle) also generally increases after PSE including events such as a half I ronman triathlon, ${ }^{9}$ a short triathlon, ${ }^{40}$ an ultra-triathlon, ${ }^{20}$ a marathon, ${ }^{38}$ a $160 \mathrm{~km}$ run, ${ }^{39}$ and a modified triathlon ( $28 \mathrm{~km}$ canoe, $90 \mathrm{~km}$ cycle, and 42.2 run). ${ }^{41}$ The increase in plasma creatinine concentration is probably the result of release of creatinine from the working muscles, dehydration, and/or a reduction in renal blood flow and glomerular filtration rate. 


\section{Take home message}

Doctors and athletes should be aware of the potential adverse biochemical changes, especially hyponatraemia, that may result from prolonged strenuous exercise.

The transient increase in creatinine and urea after strenuous exercise is thought to be of little clinical concern to renal function. However, some investigators have observed small, but significant, indices of renal damage after prolonged endurance exercise resulting from low blood flow to the kidney. ${ }^{38}{ }^{42}$ There have al so been reports of acute renal failure in some athletes after PSE. ${ }^{43}$ It still remains to be determined whether the repeated performance of prolonged endurance events leads to renal alterations that are of concern from a health point of view.

\section{CONCLUSIONS}

The performance of a prolonged endurance event is associated with several potential biochemical changes, of which it is important for athletes and doctors to be aware. Although the findings are not uniform, several investigators have shown that PSE may be associated with hypomagnesaemia, hypokalaemia, hyponatraemia, and/or increased concentrations of urea and creatinine. Adverse reactions, from a health point of view, resulting from these biochemical changes are rare. However, doctors and athletes should particularly be aware of the potentially life threatening effects of hyponatraemia and the appropriate treatment.

\section{Authors' affiliations}

D E R Warburton, R C Welsh, M J Haykowsky, D A Taylor, D P Humen, Division of Cardiology, Faculty of M edicine, University of Alberta, Edmonton, Alberta, Canada

Correspondence to: Dr W arburton, Allan McG avin Sports M edicine Centre, 3055 W esbrook Mall, University of British Columbia, Vancouver, BC V6T 1Z3, Canada; darrenwb@interchange.ubc.ca

Accepted 23 February 2002

\section{REFEREN CES}

1 O'Toole ML, Hiller W D, Roalstad MS, et al. Hemolysis during triathlon races: its relation to race distance. Med Sci Sports Exerc $1988 ; 20: 272-5$

2 Gledhill N, W arburton D, Jamnik V. Haemoglobin, blood volume, cardiac function, and aerobic power. Can J Appl Physiol $1999 ; 24: 54-65$

3 Speedy DB, N oakes TD, Rogers IR, et al. Hyponatremia in ultradistance triathletes. Med Sci Sports Exerc 1999;31:809-15.

4 Noakes TD, Norman RJ, Buck RH, et al. The incidence of hyponatremia during prolonged ultraendurance exercise. Med Sci Sports Exerc $1990 ; 22: 165-70$.

5 Resina A, Gatteschi L, Castellani W, et al. Effects of aerobic training and exercise on plasma and erythrocyte magnesium concentration. In: Kies CV, Driskell JA, eds. Sports nutrition: minerals and electrolytes. London: CRC Press; 1995:189-203.

6 Lijnen P, Hespel P, Fagard R, et al. Erythrocyte, plasma and urinary magnesium in men before and after a marathon. Eur J Appl Physiol $1988 ; 58: 252-6$

7 Rose LI, Carroll DR, Lowe SL, et al. Serum electrolyte changes after marathon running. J Appl Physiol 1970;29:449-51.

8 Franz KB, Ruddel H, Todd GL, et al. Physiologic changes during a marathon, with special reference to magnesium. J Am Coll $\mathrm{N}$ utr $1985 ; 4: 187-94$

9 Welsh R, W arburton DER, Haykowksy MJ, et al. Hematological response to the half ironman triathlon [abstract]. Med Sci Sports Exerc 1999;31:563.
10 Refsum HE, Meen HD, Stromme SB. W hole blood, serum and erythrocyte magnesium concentrations after repeated heavy exercise of long duration. Scand J Clin Lab Invest 1973;32:123-7.

11 Beller GA, Maher JT, Hartley LH, et al. Changes in serum and sweat magnesium levels during work in the heat. A viat Space Environ M ed 1975;46:709-12.

12 Stendig-Lindberg G, Shapiro Y, Epstein Y, et al. Changes in serum magnesium concentration after strenuous exercise. J Am Coll $\mathrm{N}$ utr $1987 ; 6: 35-40$

13 Golf SW, Happel O, G raef V, et al. Plasma aldosterone, cortisol and electrolyte concentrations in physical exercise after magnesium supplementation. J Clin Chem Clin Biochem 1984;22:717-21.

14 Rowe WJ. Endurance exercise and injury to the heart. Sports Med 1993;16:73-9.

15 Rowe WJ. Extraordinary unremitting endurance exercise and permanent injury to normal heart. Lancet 1992;340:712-14.

16 Chadda KD, Schult N A. Magnesium deficiency and coronary vasospasm: role in sudden cardiac death. Magnesium 1982;1:84-94.

17 Struthers AD, Q uigley C, Brown MJ. Rapid changes in plasma potassium during a game of squash. Clin Sci 1988;74:397-401.

18 Thomson A, Kelly DT. Exercise stress-induced changes in systemic arterial potassium in angina pectoris. Am J Cardiol 1989;63:1435-40.

19 Medbo JI, Sejersted O M. Acid-base and electrolyte balance after exhausting exercise in endurance-trained and sprint-trained subjects. Acta Physiol Scand 1985;125:97-109.

20 Gastmann U, Dimeo F, Huonker M, et al. Ultra-triathlon-related blood-chemical and endocrinological responses in nine athletes. J Sports Med Phys Fitness 1998;38:18-23.

21 Knochel JP, Blachley JD, Johnson JH, et al. M uscle cell electrical hyperpolarization and reduced exercise hyperkalemia in physically conditioned dogs. I Clin Invest 1985;75:740-5.

22 Ledingham IM, M acVicar S, W att I, et al. Early resuscitation afte marathon collapse [letter]. Lancet 1982;2:1096-7.

23 Hiller WD. Dehydration and hyponatremia during triathlons. Med Sci Sports Exerc 1989;21:S219-21.

24 Noakes TD. The hyponatremia of exercise. Int / Sport $N$ utr $1992 ; 2: 205-28$

25 Garigan TP, Ristedt DE. Death from hyponatremia as a result of acute water intoxication in an Army basic trainee. Mil Med 1999;164:234-8

26 Backer HD, Shopes E, Collins SL, et al. Exertional heat illness and hyponatremia in hikers. Am J Emerg Med 1999;17:532-9.

27 Montain SJ, Sawka M N , W enger CB. Hyponatremia associated with exercise: risk factors and pathogenesis. Exerc Sport Sci Rev 2001:29:113-7.

28 Hiller WD, 0 'Toole ML, Fortess EE, et al. Medical and physiological considerations in triathlons. Am J Sports Med 1987;15:164-7.

29 O'Toole ML, Douglas PS, Laird RH, et al. Fluid and electrolyte status in athletes receiving medical care at an ultradistance triathlon. Clin J Sport Med 1995;5:116-22

30 Speedy DB, N oakes TD, Kimber NE, et al. Fluid balance during and after an ironman triathlon. Clin J Sport Med 2001;11:44-50.

31 Noakes TD. Hyponatremia during endurance running: a physiological and clinical interpretation. Med Sci Sports Exerc 1992;24:403-5.

32 Frizzell RT, Lang GH, Lowance DC, et al. Hyponatremia and ultramarathon running. JAMA 1986;255:772-4

33 Noakes TD, Goodwin N, Rayner BL, et al. W ater intoxication: a possible complication during endurance exercise. Med Sci Sports Exerc $1985 ; 17: 370-5$

34 Holtzhausen LM, N oakes TD. Collapsed ultraendurance athlete: proposed mechanisms and an approach to management. Clin J Sport Med 1997;7:292-301.

35 Surgenor S, Uphold RE. Acute hyponatremia in ultra endurance athletes Am J Emerg M ed 1994;12:441-4.

36 Janssen GM, Degenaar CP, M enheere PP, et al. Plasma urea, creatinine, uric acid, albumin, and total protein concentrations before and after 15-25-, and 42-km contests. Int I Sports M ed 1989;10(suppl 3):S132-8

37 Refsum HE, Tveit B, Meen HD, et al. Serum electrolyte, fluid and acid-base balance after prolonged heavy exercise at low environmental temperature. Scand J Clin Lab Invest 1973;32:117-22.

38 Whiting PH, Maughan RJ, Miller JD. Dehydration and serum biochemical changes in marathon runners. Eur J Appl Physiol 1984;52:183-7.

39 Noakes TD, Carter JW. Biochemical parameters in athletes before and after having run 160 kilometres. S A fr M ed J 1976;50:1562-6.

40 Long $D$, Blake $M, M c N$ aughton $L$, et al. Hematological and biochemical changes during a short triathlon competition in novice triathletes. Eur J Appl Physiol 1990;61:93-9

41 van Rensburg JP, Kielblock AJ, van der Linde A. Physiologic and biochemical changes during a triathlon competition. Int J Sports $\mathrm{Med}$ $1986 ; 7: 30-5$

42 Melamed I, Romem Y, Keren G, et al. M arch myoglobinemia: a hazard to renal function. Arch Intern M ed 1982;142:1277-9.

43 MacSearraigh ET, Kallmeyer JC, Schiff HB. A cute renal failure in marathon runners. N ephron 1979;24:236-40. 